Background: Reports of rheumatic immune-related adverse events (irAEs) in patients receiving immune checkpoint inhibitors $(\mathrm{ICPi})$ have recently attracted new attention to the complex interrelations of malignancies and rheumatic and musculoskeletal diseases (RMDs). Since those two entities represent two sides of a dysregulated immune response, further research on rheumatic irAEs and mechanisms underlying the better tumor response rates in irAE-affected patients may contribute to a better understanding of the different pathophysiology characterizing tumor and rheumatic disease.

Objectives: Given the heterogeneity of the patient population with rheumatic irAEs, a registry-based study has been conducted to provide first evidence regarding characteristics of rheumatic irAEs and further insights into the optimal diagnostic and therapeutic management of rheumatic irAEs.

Methods: The TRheuMa registry is a long-term, open-end observational study of a patient cohort suffering from rheumatic symptoms as a result of ICPi or other cancer therapies. The TRheuMa registry is one of the three subregistries of the MalheuR project, a registry-based study initiated in July 2018 at the at the university hospital Heidelberg to explore interrelations of malignancies and RMDs. Results: Over 18 months, 52 of 63 patients in the TRheuMa registry were recruited with a rheumatic irAE under ICPi treatment (pembrolizumab $n=21$, nivolumab $n=28$, ipilimumab $n=11$, durvalumab $n=1$, atezolizumab $n=2$, avelumab $n=1$, history of $>1$ ICPi $n=11)$. Of the 52 patients, $22(42.3 \%)$ had non-small cell lung cancer and 23 $(44.2 \%)$ had a melanoma. Eight (15.3\%) patients experienced a flare of a preexisting RMD under ICPi treatment. The remaining 44 patients with de novo irAEs were characterized by rheumatoid arthritis-like $(20.5 \%)$ or polymyalgia rheumatica-like (18.1\%) and psoriatic or other spondyloarthritis-like phenotypes (50.0\%). However, laboratory findings differed from classical RMDs with elevated CRP-levels in $73.1 \%$ particularly in psoriatic arthritis-like, but not necessarily in polymyalgia rheumatica-like irAEs. On the contrary, autoantibody positivity was very rare. The majority of patients $(78.8 \%)$ showed signs of inflammation upon ultrasound examination.

Based on the severity of signs and symptoms as well as treatment response, we developed a therapeutic algorithm for rheumatic irAEs: non-steroidal anti-inflammatory drugs and/or low dosed glucocorticoids ( $\leq 10 \mathrm{mg}$ prednisone equivalent) as first treatment step were sufficient for $75 \%$ patients, whereas $17.3 \%$ required higher dosed glucocorticoids and $11.5 \%$ patients required further treatment with a cs- or bDMARD. In two cases ICPi-treatment was discontinued on patients' request due to the pain and functional impairment caused by the rheumatic irAE, although a satisfactory symptom control was reached in the further course.

Complete remission of cancer was observed in $43.5 \%$ of melanoma patients, $66.7 \%$ experienced additional severe irAEs in other organ systems.

Conclusion: Overall, data from the TRheuMa-registry show that rheumatic irAEs mostly resemble classical RMDs, however show distinct characteristics. Our diagnostic and therapeutic management of rheumatic irAEs demonstrated efficacy in the majority of patients. These findings contribute to the further understanding of rheumatic irAEs and malignancies. Future research agenda includes a correlation of irAE severity with tumor response.

Disclosure of Interests: Karolina Benesova Grant/research support from: Study grants for SCREENED study by Abbvie, Novartis and Rheumaliga Baden-Württemberg, Consultant of: One-time participation in Novartis advisory board., Leonore Diekmann: None declared, Hanns-Martin Lorenz Grant/research support from: Consultancy and/or speaker fees and/or travel reimbursements: Abbvie, MSD, BMS, Pfizer, Celgene, Medac, GSK, Roche, Chugai, Novartis, UCB, Janssen-Cilag, Astra-Zeneca, Lilly. Scientific support and/or educational seminars and/or clinical studies: Abbvie, MSD, BMS, Pfizer, Celgene, Medac, GSK, Roche, Chugai, Novartis, UCB, Janssen-Cilag, Astra-Zeneca, Lilly, Baxter, SOBI, Biogen, Actelion, Bayer Vital, Shire, Octapharm, Sanofi, Hexal, Mundipharm, Thermo Fisher., Consultant of: see above, Karin Jordan Consultant of: Consultancy and/or speaker fees: MSD, Merck, Amgen, Hexal, Riemser, Helsinn, Tesaro, Kreussler, Voluntis, Pfizer, Pomme-med., Jan Leipe Grant/research support from: Consultancy and speaker fees: Abbvie, AstraZeneca, BMS, Celgene, Hospira, Janssen-Cilag, LEO Pharma, Lilly, MSD, Novartis, Pfizer, Roche, Sanofi, UCB. Scientific support: Novartis, Pfizer., Consultant of: Consultancy and speaker fees: Abbvie, AstraZeneca, BMS, Celgene, Hospira, Janssen-Cilag, LEO Pharma, Lilly, MSD, Novartis, Pfizer, Roche, Sanofi, UCB. Scientific support: Novartis, Pfizer., Speakers bureau: Abbvie, AstraZeneca, BMS, Celgene, Hospira, Janssen-Cilag, LEO Pharma, Lilly, MSD, Novartis, Pfizer, Roche, Sanofi, UCB

DOI: 10.1136/annrheumdis-2020-eular.3790

\begin{tabular}{|l|l}
\hline OP0271 & REAL-WORLD CLINICAL BURDEN AND \\
GLUCOCORTICOID USE IN PATIENTS WITH \\
POLYMYALGIA RHEUMATICA
\end{tabular}

R. Punekar ${ }^{1}, \underline{\text { P. Lafontaine }}^{2}$, J. H. Stone ${ }^{3}$. 'Sanofi, Cambridge, United States of America; ${ }^{2}$ Sanofi, Bridgewater, United States of America; ${ }^{3}$ Harvard Medical School, Boston, United States of America

Background: Polymyalgia rheumatica (PMR) is a chronic inflammatory condition characterized by aching and morning stiffness in the neck, shoulders and pelvic girdle. It is a common inflammatory rheumatic disease in patients age $>50$ years, particularly women. While giant cell arteritis (GCA) is present in $9-21 \%$ of PMR cases, many PMR patients have symptoms independent of GCA. Current treatment options are limited to long-term glucocorticoid (GC), however, with risks of GC-related complications, including cardiovascular disease, osteoporosis, and diabetes mellitus.

Objectives: To compare GC use and subsequent GC-related complications in patients with PMR vs a general population $(\mathrm{GnP})$ cohort.

Methods: This retrospective, observational cohort study was based on Optum's de-identified Clinformatics ${ }^{\circledR}$ Data Mart Database (study period 01Jan200630June2018). The PMR cohort included patients with $\geq 1$ inpatient or $\geq 2$ outpatient claims $\geq 30$ days apart with PMR related diagnosis codes (ICD-9: 725 xx or ICD-10: M35.3x) between 01Jan2006-30June2017 (patient identification period) during which first occurrence of a PMR-related medical claim was set as the index date (ID). Patients with $\geq 1$ medical claim related to rheumatoid arthritis (RA) or GCA during the study period were excluded. The GnP cohort included patients without any RA, GCA or PMR diagnosis codes during the study period, with their ID set as 12 months from the start of continuous health plan enrollment Patients in both cohorts were required to be age $\geq 50$ years (on ID) with continuous health plan enrollment $\geq 12$ months pre- and post-ID. Cohorts were 1:1 propensity score matched. GC use and incidence of GC-related complications were assessed from GC initiation, starting from the baseline period (12-months pre-ID) through to the end of GC use during the post-index period (i.e. the end of data availability, end of the study period or death [whichever occurred first]) Mean, standard deviation (SD) and median values for continuous variables, and frequency ( $\mathrm{n}$ and \%) for categorical variables were compared between the matched cohorts. Wilcoxon sum rank tests and $t$-tests on continuous variables and Chi-square tests or Fisher's exact tests on categorical variables between matched cohorts were conducted. Duration of GC use was analyzed using the Kaplan-Meier method and compared between matched cohorts using log-rank tests.

Results: In each of the PMR and GnP cohorts, 16,865 patients were included. In both matched cohorts, median age was 76 years, median Elixhauser comor bidity index score was 2.0 , and the majority ( $65 \%$ ) were women. The median follow-up duration was 45 months and 51 months in the PMR and GnP cohorts respectively. A higher proportion of patients in the PMR cohort than the matched GnP cohort ( $90.4 \%$ vs $62.8 \% ; p<0.001)$ used GC. The mean (SD) duration of GC therapy was significantly longer in the PMR cohort than in the matched GnP cohort $(242.1[ \pm 317.2]$ days vs $35.5[ \pm 124.6]$ days; $p<0.001)$. Although patients in the PMR cohort had a lower average daily dose of GC (prednisone equivalent) vs the GnP cohort (mean [SD] mg $16.3[ \pm 21.9]$ vs $27.8[ \pm 24.5]$, respectively $[p<0.0001)]$, the cumulative $\mathrm{GC}$ dose was significantly higher in the PMR cohort than the GnP cohort $(2125.4[ \pm 3689.5] \mathrm{mg}$ vs $476.6[ \pm 1450.9] \mathrm{mg} ; p<0.001)$. This indicates PMR patients used chronic low dose GC while the GnP patients utilized higher dose GC burst therapy less frequently. The number of incident complications associated with GC use were significantly greater in the PMR cohort, and included hypertension, diabetes, skin toxicity, infections, neuropsychiatric effects, endocrine abnormalities, renal dysfunction/ failure, ocular effects, and cardiovascular disease $(p<0.05)$

Conclusion: The overall GC burden in patients with PMR is high. With a higher incidence of GC-related comorbidities among PMR patients, early onset of these complications may be a significant contributor to long-term healthcare costs in these patients.

Acknowledgments: This study was funded by Sanofi, Inc. Medical writing under the direction of authors, was provided by Gauri Saal, MA Economics, Prime, Knutsford, UK, and funded by Sanofi.

Disclosure of Interests: Rajeshwari Punekar Shareholder of: Sanofi, Employee of: Sanofi, Patrick LaFontaine Shareholder of: Sanofi, Employee of: Sanofi, John H. Stone Grant/research support from: Roche, Consultant of: Roche DOI: 10.1136/annrheumdis-2020-eular.4285

\section{OP0272 LONG-TERM EFFICACY AND SAFETY OF CANAKINUMAB IN PATIENTS WITH COLCHICINE- RESISTANT FAMILIAL MEDITERRANEAN FEVER: RESULTS FROM THE RANDOMISED PHASE 3 CLUSTER TRIAL}

S. Özen ${ }^{1}$, E. Ben-Chetrit ${ }^{2}$, I. Foeldvari ${ }^{3}$, G. Amarilyo ${ }^{4}$, H. Ozdogan ${ }^{5}$, S. Vanderschueren ${ }^{6}$, K. Marzan ${ }^{7}$, J. M. Kahlenberg ${ }^{8}$, E. Dekker ${ }^{9}$, F. De Benedetti ${ }^{10}$, I. Koné-Paut ${ }^{11}$. ${ }^{1}$ Hacettepe University, Department of Pediatric Rheumatology, Ankara, Turkey; ${ }^{2}$ Hadassah-Hebrew University Medical Center, Rheumatology Unit, Jerusalem, Israel; ${ }^{3} \mathrm{Hamburg}$ Centre for Pediatric and Adolescent Rheumatology, An der Schön Klinik, Hamburg, Germany; ${ }^{4}$ Schneider Children's Medical Center of Israel, Tel Aviv, Israel; ${ }^{5}$ University of Istanbul-Cerrahpaşa, Department of Medicine, Istanbul, Turkey; ${ }^{6}$ University Hospitals Leuven, Leuven, Belgium; ${ }^{7}$ Children's Hospital Los Angeles, Los Angeles, United States of America; ${ }^{8}$ University of Michigan, Department of 